\title{
Phosphorylated T567 ezrin is associated with merlin expression in KIT-mutant gastrointestinal stromal tumors
}

\author{
WEN-HUI WENG ${ }^{1,2^{*}}$, CHUN-NAN YEH $^{3 *}$, YUNG-FENG CHENG $^{1}$, GOVINDA LENKA $^{2}$ and YI-WEI LIAW ${ }^{1}$ \\ ${ }^{1}$ Department of Chemical Engineering and Biotechnology, Graduate Institute of Biotechnology; ${ }^{2}$ College of Engineering, \\ Energy and Optoelectronic Materials Program, National Taipei University of Technology, Taipei; \\ ${ }^{3}$ Department of Surgery, Chang Gung Memorial Hospital, Chang Gung University, Taoyuan, Taiwan, R.O.C.
}

Received June 8, 2011; Accepted September 13, 2011

DOI: $10.3892 / \mathrm{mmr} .2011 .609$

\begin{abstract}
Membrane-cytoskeleton linker organizer ezrin is a member of the ERM (ezrin-radixin-moesin) protein family. It has been suggested as an important element in the oncogenic process, particularly in conferring a metastatic ability on tumor cells. We hypothesized that the KIT oncogenic form is one of the proteins that modulates expression of the ezrin protein via phosphorylated ezrin at different residues; furthermore, it may interact with the protein merlin, and promoting tumor development via the PI3K or MAPK pathway. In the present study, we observed that differential expression of ezrin was a common feature in gastrointestinal stromal tumors (GISTs). We further demonstrated that cases exhibiting expression of phosphorylated Thr567 in the ezrin protein were associated with immunoactivities of KIT and merlin expression ( $\mathrm{p}=0.039$ and 0.013 , respectively). In conclusion, GISTs harbor activation of KIT protein may induce phosphorylation of the downstream protein ezrin at certain residues, thereby triggering subsequent signal transduction cascades and driving downstream pathways of tumor progression. However, a larger series of tumor samples should be analyzed in future studies, as well as the identification of phosphorylated sites to determine the role of ezrin in tumor progression thus shedding light on clinical outcomes.
\end{abstract}

\section{Introduction}

Investigations spanning almost a decade reveal that the membrane-cytoskeleton linker protein ezrin plays an important role in promoting tumor metastasis $(1,2)$. It is therefore

Correspondence to: Dr Wen-Hui Weng, Department of Chemical Engineering and Biotechnology, Graduate Institute of Biotechnology, National Taipei University of Technology, 1, Sec. 3, Chung-Hsiao E. Rd., Taipei 10608, Taiwan, R.O.C.

E-mail:wwhlab@gmail.com

*Contributed equally

Key words: ezrin, KIT, merlin, p-ezrinThr567, p-ezrinTyr146, p-ezrinTyr353 generally considered to be one of the predictive prognostic biomarkers in various cancer types, including osteosarcoma, breast cancer, colorectal carcinoma, soft tissue sarcoma and serous ovarian carcinoma (3-10). Ezrin protein is a member of the ERM (ezrin-radixin-moesin) group of proteins that are produced from the VIL2 (Ctytovillin) gene, which is a cyclic AMP-dependent protein kinase anchoring protein. Activation of ezrin is known to be caused by phosphorylation at certain residues, in turn interacting directly with the actin by the C-terminus, and connecting with several transmembrane proteins or membrane-associated partners via the aminoterminal FERM (four-point-one, ezrin, radixin, moesin) domain (11). There are reports indicating that the functions of ezrin particularly operate in the regulation of epithelial cell morphogenesis, cell-cell and cell-matrix adhesion proceed through various pathways (12). Furthermore, the unexpected phosphorylation at certain residues of ezrin is vital for tumor progression. Monni et al observed that phosphorylated ezrin at the Tyr353 and Tyr146 residues may induce tumor cell apoptosis and promote cell proliferation in murine erythroleukemia, respectively (13). In addition, the binding of phosphatidylinositol 4,5-bisphosphate (PIP2) with ezrin N-terminal ERM association domain is necessary for the subsequent phosphorylation at Thr567, which is involved in the subsequent activation process to unmask both membrane and actin binding sites (14), which then extend to interact with CD43 and CD44 (15-18). Moreover, some of the studies further indicated that a mutant form of ezrin by mimic a phosphorylated residue ezrin T567D may maintain the protein in an open conformation that will further trigger the activity of the Rac1 pathway (but not RhoA or Cdc 42) $(19,20)$.

Conversely, merlin acts as an inhibitor of small G-protein activation, a role more like a 'gatekeeper', in many types of tumors. The protein merlin is encoded by the tumor-suppressor gene Neurofibromin 2 (NF2), from which multiple isoforms are generated after transcripts undergo alternative splicing. However, only isoform I functions as a tumor-suppressor protein, and the activitity is according to the phosphorylated status $(21,22)$. The location of the merlin protein in cells is rather similar to ezrin. They are commonly present at the membrane-cytoskeleton interface underneath the plasma membrane, cell-cell junctions, as well as actinrich sites (23); thus the structure of merlin shares N-terminal 
sequence homology with the ERM protein family, except that it lacks the actin binding region. The behavior of merlin is extremely opposite to ezrin proteins, while phosphorylated-open forms exhibit loss of the function of the protein. Therefore, to decide whether the merlin or ezrin protein binds to the transmembrane proteins, such as CD44, depends on their status of phosphorylation. According to a report from Ponta et al, merlin negatively regulates $\mathrm{RAC1}$; it is considered to be a protein which competes with the function of ezrin (11).

Based on Monni et al, ezrin protein may be a downstream target of the KIT gene, a receptor tyrosine kinase (RTK) (13). Other studies have also provided strong evidence regarding the possible mechanism of the mutant KIT, and have all indicated that it may affect the downstream signaling transduction; to regulate the activation of ezrin may be one of the possibilities, which eventually results in cell proliferation, anti-apoptosis or induction of cell migration. GISTs is one of the diseases related to KIT expression, which is characterized by the presence of KIT (CD117) protein activation in 75-90\% of cases (24-33). Therefore, in the present study, we hypothesized that oncogenic KIT mutations may regulate ezrin and/or merlin expression in human GIST cells. In addition, the status of phosphorylated ezrin at different residues may offer a valuable piece of information in KIT-related tumor progression.

\section{Materials and methods}

Clinical samples and cell lines. A total of 13 fresh frozen tumor samples, characterized as GISTs, were kindly provided by Dr Chun-Nan Yeh of the Surgical Department of Chang Gung Memorial Hospital, Taiwan. All clinical samples had the approval of the research ethics committee and informed patient consent. The human c-KIT-positive GIST882 and c-KIT-negative GIST62 cell lines were also included as internal controls for detection of KIT expression. It is known that the GIST882 cell line with a homozygous exon 13 missense mutation, encoding a K642E results in KIT expression (34); the GIST62 cell line used to harbor an in frame exon 11 mutation after passage subsequently loses KIT expression (35). Both cell lines were grown in RPMI-1640 (Invitrogen, Carlsbad, CA, USA) containing 20\% FBS. The A431 human epithelial carcinoma and human embryonic kidney 293 (HEK293) cell lines were used as positive controls for detection of ezrin or merlin protein expression, respectively. It is known that the A431 cell line contains phosphorylated p81-ezrin on serine and threonine residues. However, after treatment with 100 ng/ml EGF p81-ezrin may become phosphorylated on tyrosine or phosphorylation of threonine residues increases $(36,37)$.

Protein extraction. The lysate preparation of fresh tumor samples and cell line samples was carried out for the Western blot analysis. Fresh tissue samples were intially maintained at a low temperature to homogenize the tissues before being washed 3 times with ice-cold PBS, and then lysed in Pro-Prep ${ }^{\mathrm{TM}}$ protein extraction solution (iNtRON Biotechnology Inc., Seongnam, Korea) containing phosphatase inhibitor cocktail (Thermo Scientific, USA) according to the manufacturer's instructions (iNtRON Biotechnology Inc.). In brief, to induce cell lysis, the cells were incubated for 10-30 min on ice, followed by centrifugation at $13,000 \mathrm{rpm}$, at $4^{\circ} \mathrm{C}$ for $5 \mathrm{~min}$ then stored at $-20^{\circ} \mathrm{C}$. The concentration of protein was determined using the Bradford method and detected by operating the Thermo Scientific NanoDrop 2000 protein assay.

Western blot assays. All of the protein samples were resolved by $8-10 \%$ SDS-polyacrylamide gel electrophoresis and then transferred to PVDF membranes (Millipore Corporation, Billerica, MA, USA). Western blotting was performed with anti-ezrin (mouse monoclonal antibody, 1:300 dilution) (Lab Vision Corp, Fremont, CA, USA.), anti-phospho-ezrin (Tyr353) (rabbit polyclonal antibody, 1:1000 dilution) (Cell Signaling Technology, Inc., Danvers, MA, USA), antiphospho-ezrin (Tyr146) (rabbit polyclonal antibody, 1:400 dilution) (Santa Cruz Biotechnology Inc., Santa Cruz, CA, USA) anti-phospho-ezrin (Thr567)/Radixin (Thr564)/ Moesin (Thr558) (41A3) (rabbit monoclonal antibody, 1:1000 dilution) (Cell Signaling Technology Inc.), anti-Kit (C-19) (rabbit polyclonal antibody, 1:300 dilution) (Santa Cruz Biotechnology Inc.), and anti-NF2 (A-19) (rabbit polyclonal antibody, 1:300 dilution) (Santa Cruz Biotechnology Inc.) antibodies. $\beta$-actin antibody was used as the loading control (Novus Biologicals, Littleton, CO, USA) for each set of experiments.

Statistical analysis. To investigate the correlation between the protein expression of KIT and the status of phosphorylated ezrin at the Thr567, Tyr146 and Tyr353 residues, as well as merlin expression, the samples were divided into KIT-positive (10 cases) or KIT-negative (3 cases) groups to correlate with the other proteins as shown in Table I. The Chi-square test was performed, and P-values $<0.05$ were considered to denote statistical significance.

\section{Results}

Western blot analysis. In the present study, Western blot analysis was performed to detect protein expression of KIT, total ezrin, phosphorylated status at ezrin Thr567, Tyr146, Tyr353 residues, and merlin. The results showed that 10 out of $13(77 \%)$ GIST samples exhibited KIT expression. In addition, ezrin protein was expressed at different levels in all of the samples (100\%) (Table I). Particularly, case no. 5 exhibited high expression when compared to the others (Fig. 1). Notably, when comparing the different residues Thr567, Tyr146 and Tyr353 and expression of phosphorylated ezrin, the high percentage of cases showed phosphorylation of ezrin at the Thr567 residue (85\%). Interestingly, merlin expression was commonly observed in the tumors that simultaneously expressed both KIT and ezrin proteins (cases 3-5) (Fig. 1).

Chi-square test analysis. To investigate the correlation between the expression of KIT and the status of phosphorylated ezrin at the different residues, as well as merlin in the GIST samples, the Chi-square test showed KIT expression in the GIST cases was highly associated with phosphorylated ezrin at Thr567 or merlin expression (both $\mathrm{P}=0.039$ ). Notably, all of the cases that contained phosphorylated ezrin at Tyr146 also exhibited expression of merlin (Table I). 
Table I. Correlation between the expression of KIT and different phosphorylation sites of ezrin and merlin in GISTs.

\begin{tabular}{|c|c|c|c|c|c|c|c|}
\hline \multirow[t]{3}{*}{ Protein } & \multicolumn{6}{|c|}{ GISTs (n) } & \multirow[t]{3}{*}{ Rate of expression (\%) } \\
\hline & \multicolumn{2}{|c|}{ KIT } & \multirow[t]{2}{*}{ P-value } & \multicolumn{2}{|c|}{ Merlin } & \multirow{2}{*}{ P-value } & \\
\hline & + & - & & + & - & & \\
\hline \multicolumn{8}{|l|}{ Ezrin } \\
\hline Expressed & 10 & 3 & - & 11 & 2 & - & 100 \\
\hline \multicolumn{8}{|l|}{ p-ezrinThr567 } \\
\hline Expressed & 10 & 1 & 0.039 & 11 & 0 & 0.013 & 85 \\
\hline Not expressed & 0 & 2 & & 0 & 2 & & 15 \\
\hline \multicolumn{8}{|l|}{ p-ezrinTyr146 } \\
\hline Expressed & 5 & 0 & 0.231 & 5 & 0 & 0.487 & 39 \\
\hline Not expressed & 5 & 3 & & 6 & 2 & & 61 \\
\hline \multicolumn{8}{|l|}{ p-ezrinTyr353 } \\
\hline Expressed & 4 & 1 & 0.835 & 5 & 0 & 0.487 & 39 \\
\hline Not expressed & 6 & 2 & & 6 & 2 & & 61 \\
\hline \multicolumn{8}{|l|}{ Merlin } \\
\hline Expressed & 10 & 1 & 0.039 & & & & 85 \\
\hline Not expressed & 0 & 2 & & & & & 15 \\
\hline
\end{tabular}

$\mathrm{n}$, the number of cases; +, positive expression of protein; -, negative expression of protein; P-values $<0.05$ are indicated in bold.
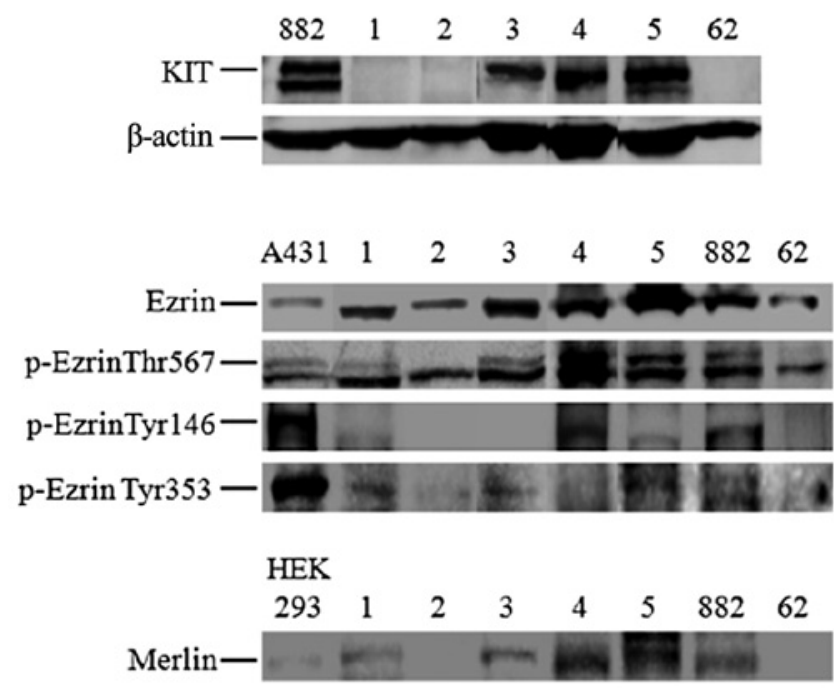

Figure 1. Western blot analysis showed the expression of KIT, p-ezrinThr567, p-ezrinTyr146, p-ezrinTyr354 and merlin in the GIST samples. GIST882 and GIST62 cell lines were used as KIT-positive and -negative internal controls, respectively. A431 and HEK293 cell lines were used as the positive controls to detect expression of total ezrin or phosphorylated ezrin and merlin, respectively. B-actin served as the loading control.

\section{Discussion}

In the present study, different expression levels of ezrin total protein were observed in all samples (Table I). A high correlation between the expression of KIT and phosphorylated ezrin at Thr567 or merlin was noted $(\mathrm{P}=0.039$ and 0.013 , respectively). A recent study provided evidence

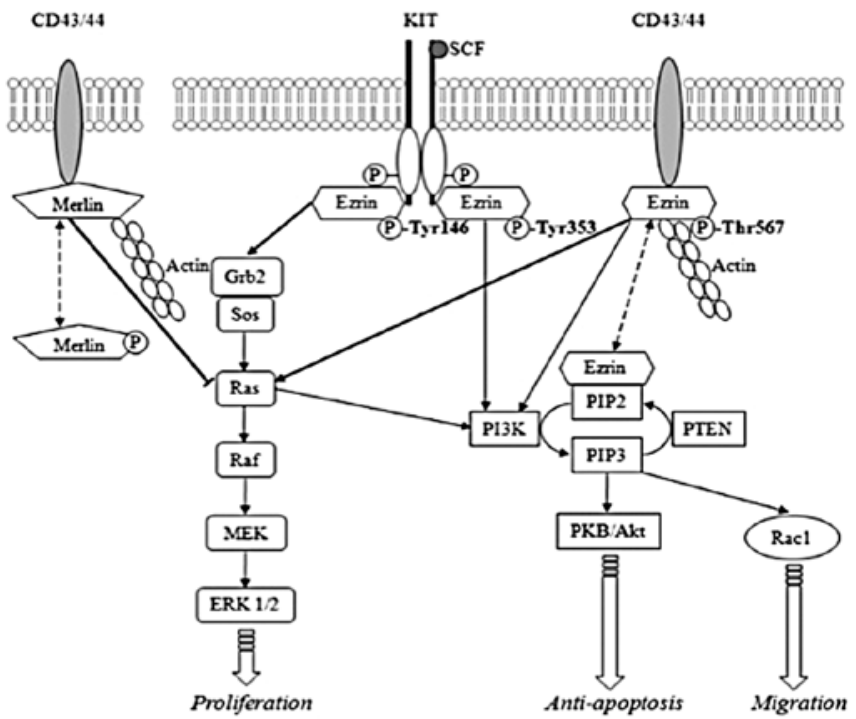

Figure 2. Schematic illustration of the proposed mechanism of ezrin protein phosphorylation upon the activation of (mutant) KIT. KIT ligand/receptor activity induces phosphorylation of ezrin at Tyr146 and Tyr353, which exerts RAS/MAPK and PI3K signaling transduction pathways. Hence, p-ezrinThr567 indirectly affects RAC1 activation through the PI3K pathway. Whereas merlin was presented as a negative regulated form, p-ezrinTyr146 and p-ezrinThr567 were triggered and mediated the RAS/MAPK pathway as indicated by bold arrows.

that ezrin overexpression in GISTs may be an independent adverse prognostic indicator (29). Hence, in several types of cancers such as sarcoma, mesenchymal tissue tumors, hepatocellular carcinoma and prostate cancer, ezrin was found to play an important role in tumor progression and was highly 
associated with tumor metastasis $(9,38,39)$. Monni et al found that phosphorylated ezrin at certain residues, for example, p-ezrinTyr146 and p-ezrinTyr353, acted as a downstream effector of oncogenic tyrosine kinases in a study of murine erythroleukemia (13). Moreover, KIT ligand/stem cell factor (SCF) may induce phosphorylation on threonine residue of ERM proteins, besides, the increasing of Racl activation through the activation of PI3K was also demonstrated in human melanocytes (40). Accordingly, we proposed that ezrin is an important target to further drive tumor progression, particularly in tumors associated with oncogenic KIT mutants, such as GISTs.

Thus, in the present study, a total of 13 clinical GIST cases were investigated, and 10 cases confirmed to be KIT-positive were included. Expression of p-ezrinThr567 and merlin were simultaneously present in the KIT-positive GIST samples $(\mathrm{P}=0.039)$. We presumed that KIT-related tumors may simultaneously express both ezrin and merlin, although they have an opposite function as cell proteins. The underlying variant pathways may rely on KIT activation; in turn ezrin activity is regulated by the turnover of phosphorylation on the Thr567 residue in ezrin protein. This mechanism may play a key role in triggering downstream pathway activities. This finding was supported by Di Cristofano $e t$ al and Cui et al who evaluated p-ezrinTyr353 and p-ezrinThr567 expression in osteosarcoma and pancreatic ductal adenocarcinoma, respectively, and pinpointed that p-ezrinThr567 expression could be observed mostly in tumors and related to tumor progression. However, there is no strong evidence supporting the promotion of tumor metastatis $(41,42)$.

Moreover, we further observed that the cases exhibiting expression of p-ezrinTyr146, which were all associated with the expression of p-ezrinThr567. To our knowledge, the potential mechanism of tumoral development regarding p-ezrinTyr146, may be via the MEK pathway cascade, in turn promoting tumor cell proliferation (Fig. 2). Similar evidence has been provided in leukemic cells in both in vitro and in vivo studies (13). Thus, we presumed that while the tumors presented phosphorylation at both the Tyr146 and Thr567 ezrin protein residues, tumor development might be through the MEK signaling pathway promoting tumor cell proliferation. On the other hand, for the tumors exhibiting phophorylation at Thr567 of the ezrin protein only, the later signaling transduction might guide tumor cells to the consequences of anti-apoptosis or tumor migration via the PI3K pathway (Fig. 2). Accordingly, we concluded that tumor progression of KIT-related tumors, such as GISTs, strongly relies on the activities of phosphorylated ezrin residues, and the consequent activities of the ezrin protein may provide a novel clinical application.

Regarding the correlation between ezrin and merlin, due to the highly similar structure of the proteins, merlin shares its domain organization of NH2-terminal sequence homology with ERM proteins, but does not contain a canonical actinbinding motif at its $\mathrm{C}$-terminus. Thus, the phosphorylated merlin protein structure presumably opens and forms heterodimers with ezrin or other ERM proteins, and localizes at the cell cortex. Therefore, all of these may bind to identical or similar proteins of the plasma membrane (43). Once merlin protein is unable to bind either upstream of the SOS or downstream of the Ras and Rac, this may lead to cell proliferation or transformation $(23,44)$. Our present study demonstrated that the merlin-positive cases were specifically associated with p-ezrinThr567 in GISTs $(\mathrm{P}=0.013)$, but were not correlated with p-ezrinTyr353 and p-ezrinTyr146 expression. Thus, we speculated that the p-ezrinThr567 residue may play a crucial role in the interaction with merlin, and then directly modulates RAS protein activity further triggering the downstream MEK signaling transduction pathway (Fig. 2). Since one of the functions of merlin is to promote PDGFR degradation, it appears to suppress the activation of the MAPK and PI3K signaling pathways (46). The loss of function of the merlin protein in tumors may result by either phosphorylation or formed deficiency of the truncated protein that further leads to tumor progression (45). Although all of our samples presented ezrin total protein expression, we then further determined that the phosphorylated ezrin residue site was correlated with merlin expression. The corresponding phosphorylated status of merlin, or the gene structure of merlin may need to be confirmed in future studies as well.

In summary, further investigation with different phosphorylation sites to determine the role of ezrin in tumor progression is needed, and larger series of tumor samples are necessary in future studies. The results may shed light on clinical outcome.

\section{Acknowledgements}

This work was supported by grants from the National Science Council, Taipei, Taiwan, NSC 98-2314-B-027-001 and NTUT-MMH-100-14. The authors would like to thank Jia-Yi Chen and Yeu-Jye Pang for assistance in the formatting of the manuscript.

\section{References}

1. Khanna C, Wan X, Bose S, et al: The membrane-cytoskeleton linker ezrin is necessary for osteosarcoma metastasis. Nat Med 10: 182-186, 2004.

2. Yu Y, Khan J, Khanna C, et al: Expression profiling identifies the cytoskeletal organizer ezrin and the developmental homeoprotein Six-1 as key metastatic regulators. Nat Med 10: 175-181, 2004.

3. Kim C, Shin E, Hong S, et al: Clinical value of ezrin expression in primary osteosarcoma. Cancer Res Treat 41: 138-144, 2009.

4. Xu-Dong S,Zan S, Shui-er Z, et al: Expression of Ezrin correlates with lung metastasis in Chinese patients with osteosarcoma. Clin Invest Med 32: E180-188, 2009.

5. Park HR, Cabrini RL, Araujo ES, et al: Expression of ezrin and metastatic tumor antigen in osteosarcomas of the jaw. Tumori 95: 81-86, 2009.

6. Elliott BE, Meens JA, SenGupta SK, et al: The membrane cytoskeletal crosslinker ezrin is required for metastasis of breast carcinoma cells. Breast Cancer Res 7: R365-R373, 2005.

7. Li Q, Wu M, Wang H, et al: Ezrin silencing by small hairpin RNA reverses metastatic behaviors of human breast cancer cells. Cancer Lett 261: 55-63, 2008.

8. Nestl A, Von Stein OD, Zatloukal K, et al: Gene expression patterns associated with the metastatic phenotype in rodent and human tumors. Cancer Res 61: 1569-1577, 2001.

9. Weng WH, Ahlen J, Astrom K, et al: Prognostic impact of immunohistochemical expression of ezrin in highly malignant soft tissue sarcomas. Clin Cancer Res 11: 6198-6204, 2005.

10. Moilanen J, Lassus H, Leminen A, et al: Ezrin immunoreactivity in relation to survival in serous ovarian carcinoma patients. Gynecol Oncol 90: 273-281, 2003.

11. Ponta H, Sherman L and Herrlich PA: CD44: from adhesion molecules to signalling regulators. Nat Rev Mol Cell Biol 4: 33-45, 2003.

12. Takeuchi K, Kawashima A, Nagafuchi A, et al: Structural diversity of band 4.1 superfamily members. J Cell Sci 107: 19211928, 1994 
13. Monni R, Haddaoui L, Naba A, et al: Ezrin is a target for oncogenic Kit mutants in murine erythroleukemia. Blood 111 3163-3172, 2008.

14. Fievet BT, Gautreau A, Roy C, et al: Phosphoinositide binding and phosphorylation act sequentially in the activation mechanism of ezrin. J Cell Biol 164: 653-659, 2004.

15. Orian-Rousseau V, Chen L, Sleeman JP, et al: CD44 is required for two consecutive steps in HGF/c-Met signaling. Genes Dev 16 : 3074-3086, 2002.

16. Martin TA, Harrison G, Mansel RE, et al: The role of the CD44/ ezrin complex in cancer metastasis. Crit Rev Oncol Hematol 46: 165-186, 2003.

17. Kansas GS, Muirhead MJ and Dailey MO: Expression of the CD11/CD18, leukocyte adhesion molecule 1, and CD44 adhesion molecules during normal myeloid and erythroid differentiation in humans. Blood 76: 2483-2492, 1990.

18. Legras S, Gunthert U, Stauder R, et al: A strong expression of CD44-6v correlates with shorter survival of patients with acute myeloid leukemia. Blood 91: 3401-3413, 1998.

19. Pujuguet P, Del Maestro L, Gautreau A, et al: Ezrin regulates E-cadherin-dependent adherens junction assembly through Racl activation. Mol Biol Cell 14: 2181-2191, 2003.

20. Dard N, Louvet-Vallee S, Santa-Maria A, et al: Phosphorylation of ezrin on threonine T567 plays a crucial role during compaction in the mouse early embryo. Dev Biol 271: 87-97, 2004.

21. Sherman L, Jacoby LB, Lampe J, et al: CD44 expression is aberrant in benign Schwann cell tumors possessing mutations in the neurofibromatosis type 2, but not type 1, gene. Cancer Res 57: 4889-4897, 1997.

22. Gutmann DH, Geist RT, Xu H, et al: Defects in neurofibromatosis 2 protein function can arise at multiple levels. Hum Mol Genet 7 : 335-345,1998.

23. Okada T, You L and Giancotti FG: Shedding light on Merlin's wizardry. Trends Cell Biol 17: 222-229, 2007.

24. Agaram NP, Besmer P, Wong GC, et al: Pathologic and molecular heterogeneity in imatinib-stable or imatinib-responsive gastrointestinal stromal tumors. Clin Cancer Res 13: 170-181, 2007.

25. Stamatakos M, Douzinas E, Stefanaki C, et al: Gastrointestinal stromal tumor. World J Surg Oncol 7: 61, 2009.

26. Heinrich MC, Rubin BP, Longley BJ, et al: Biology and genetic aspects of gastrointestinal stromal tumors: KIT activation and cytogenetic alterations. Hum Pathol 33: 484-495, 2002.

27. Corless CL, Fletcher JA and Heinrich MC: Biology of gastrointestinal stromal tumors. J Clin Oncol 22: 3813-3825, 2004.

28. Heinrich MC, Corless CL, Duensing A, et al: PDGFRA activating mutations in gastrointestinal stromal tumors. Science 299: 708-710, 2003

29. Wei YC, Li CF, Yu SC, et al: Ezrin overexpression in gastrointestinal stromal tumors: an independent adverse prognosticator associated with the non-gastric location. Mod Pathol 22: 1351-1360, 2009

30. Pan CC, Chen PC and Chiang H: Overexpression of KIT (CD117) in chromophobe renal cell carcinoma and renal oncocytoma. Am J Clin Pathol 121: 878-883, 2004.
31. Stec R, Grala B, Maczewski M, et al: Chromophobe renal cell cancer--review of the literature and potential methods of treating metastatic disease. J Exp Clin Cancer Res 28: 134, 2009.

32. Yamazaki K, Sakamoto M, Ohta T, et al: Overexpression of KIT in chromophobe renal cell carcinoma. Oncogene 22: 847-852, 2003.

33. Lin ZH, Han EM, Lee ES, et al: A distinct expression pattern and point mutation of c-kit in papillary renal cell carcinomas. Mod Pathol 17: 611-616, 2004.

34. Tuveson DA, Willis NA, Jacks T, et al: STI571 inactivation of the gastrointestinal stromal tumor c-KIT oncoprotein: biological and clinical implications. Oncogene 20: 5054-5058, 2001.

35. Bauer S, Yu LK, Demetri GD, et al: Heat shock protein 90 inhibition in imatinib-resistant gastrointestinal stromal tumor. Cancer Res 66: 9153-9161, 2006.

36. Gould KL, Cooper JA, Bretscher A, et al: The protein-tyrosine kinase substrate, $\mathrm{p} 81$, is homologous to a chicken microvillar core protein. J Cell Biol 102: 660-669, 1986.

37. Lee JY, Moon HJ, Lee WK, et al: Merlin facilitates ubiquitination and degradation of transactivation-responsive RNA-binding protein. Oncogene 25: 1143-1152, 2006

38. Yeh CN, Pang ST, Chen TW, et al: Expression of ezrin is associated with invasion and dedifferentiation of hepatitis B related hepatocellular carcinoma. BMC Cancer 9: 233, 2009.

39. Valdman A, Fang X, Pang ST, et al: Ezrin expression in prostate cancer and benign prostatic tissue. Eur Urol 48: 852-857, 2005.

40. Jeon S, Kim NH, Kim JY, et al: Stem cell factor induces ERM protein phosphorylation through PI3K activation to mediate melanocyte proliferation and migration. Pigment Cell Melanoma Res 22: 77-85, 2009.

41. Di Cristofano C, Leopizzi M, Miraglia A, et al: Phosphorylated ezrin is located in the nucleus of the osteosarcoma cell. Mod Pathol 23: 1012-1020, 2010.

42. Cui Y, Li T, Zhang, et al: Expression of Ezrin and phosphorylated Ezrin (pEzrin) in pancreatic ductal adenocarcinoma. Cancer Invest 28: 242-247, 2010.

43. Legg JW and Isacke CM: Identification and functional analysis of the ezrin-binding site in the hyaluronan receptor, CD44. Curr Biol 8: 705-708, 1998

44. Morrison H, Sperka T, Manent J, et al: Merlin/neurofibromatosis type 2 suppresses growth by inhibiting the activation of Ras and Rac. Cancer Res 67: 520-527, 2007.

45. Thurneysen C, Opitz I, Kurtz S, et al: Functional inactivation of NF2/merlin in human mesothelioma. Lung Cancer 64: 140-147, 2009.

46. Fraenzer JT, Pan H, Minimo L Jr, et al: Overexpression of the NF2 gene inhibits schwannoma cell proliferation through promoting PDGFR degradation. Int J Oncol 23: 1493-1500, 2003. 